\title{
Siembra a través de kilómetros
}

\section{Sowing for Miles}

Susan Camila Sandoval Echavarría ${ }^{6}$ Fabián Esteban Bernal Blanco y Johan Sebastian Castillo Melo ${ }^{8}$

\section{Resumen}

El presente trabajo contiene información sobre la implementación de una carrera atlética por parte de los estudiantes de Entrenamiento deportivo de la Fundación Universitaria del Área Andina, con el fin de generar tanto la masiva integración de toda la comunidad de dicha universidad, así como una pequeña contribución para mejorar los problemas que se desencadenan debido al cambio climático en todo el mundo.

La carrera se realiza con el fin de plantar árboles en la ciudad de Bogotá, y de esta manera ayudar a que los bosques y la fauna recuperen un poco del espacio que la ciudad con sus diferentes construcciones, edificaciones y urbanizaciones le han venido robando en los últimos años. El planteamiento anterior se realiza, basados en la importancia que tienen los árboles en la vida del ser humano, tales como: brindar oxígeno, reducir el efecto invernadero, ser refugio de muchas especies animales, entre otros.

Para poder sustentar, verificar e implementar el presente proyecto fue necesario realizar y crear diferentes espacios de recolección de información tales como: investigación de aspectos y características más relevantes del cambio climático, trabajos realizados anteriormente como guía complementaria y búsqueda de posibles fundaciones aliadas para poder crear el espacio y los elementos necesarios para una siembra masiva de árboles. Por otro lado se implementó una encuesta en la población del área andina para recolectar información y comprobar que el proyecto fuese viable y atractivo para toda la comunidad.

\footnotetext{
6 Estudiante Profesional de Entrenamiento Deportivo, Fundación Universitaria del Área Andina.

Correo: ssandoval11destudiantes.areandina.edu.co

7 Estudiante Profesional de Entrenamiento Deportivo, Fundación Universitaria del Área Andina.

Correo: fbernal12Restudiantes.areandina.edu.co

8 Estudiante Profesional de Entrenamiento Deportivo, Fundación Universitaria del Área Andina.

Correo: jcastillo80Restudiantes.areandina.edu.co
} 
Palabras clave: cambio climático, carrera atlética, material reciclable, efecto invernadero, sembrar.

\section{Abstract}

This paper contains information on implementing a race by sports training students of Fundación Universitaria del Área Andina to integrate the university community massively and contribute to improving the problems triggered by climate change around the world. This race aims to plant trees in Bogotá and help forests and fauna recover some of the space that the city has been stealing from them with its constructions, buildings, and urbanizations in recent years. This approach is based on the importance of trees for human life, such as providing oxygen, reducing the greenhouse effect, and housing many animal species. It was necessary to collect data in stages to support, verify, and implement this project, such as investigating the most relevant aspects and characteristics of climate change, searching for previous studies as a complementary guide, and partnering with foundations to provide the space and elements necessary for massive tree planting. Moreover, a survey was conducted with the university population to gather information and confirm whether the project was viable and attractive for the entire community.

Keywords: Climate change, Race, Recyclable material, Greenhouse effect, To sow.

\section{Introducción}

En la actualidad el cambio climático está generando gran preocupación en todo el mundo. Cada día es más notorio el desgaste que la humanidad le está produciendo al planeta por medio de la contaminación ambiental, los procesos industriales y el aprovechamiento de manera desmedida de los recursos naturales, como es la tala de árboles. Hoy en día la población ha hecho de esta ac- tividad un negocio muy grande, donde se busca enriquecer los bolsillos de las grandes industrias sin tener en cuenta las graves consecuencias ambientales que se generan. Es inevitable que tantos excesos y despreocupaciones por el cuidado del planeta no desencadenaran en los problemas actuales.

Los árboles son la fuente número uno de vida del planeta. Sin estos, no habría H20 ni se podría nivelar el efec- 
to invernadero. Siguiendo a Bellever (2018) quien expone que "Los distintos tipos de árboles ayudan a prevenir la erosión del suelo en zonas con laderas o pendientes", los árboles contribuyen a mantener el suelo en su lugar y mantienen la humedad del mismo gracias a sus raíces y sedimentos. Por otro lado, Panadero (2010) sustenta que "Bajo la copa de los árboles se reduce entre 2 y $9^{\circ}$ $\mathrm{C}$ la temperatura con relación a las áreas de potrero abierto". Es importante tener un campo cubierto por la sombra de los árboles, pues permiten el descanso, la nivelación de la temperatura corporal y brindan oxígeno saludable a todos los seres vivos. Finalmente, siguiendo a Corzo (2007) "Los árboles contribuyen a disminuir la contaminación de todo tipo presente en las ciudades, ciertas especies pueden absorber contaminantes del aire como el dióxido de carbono (CO2)".

Teniendo en cuenta lo expuesto anteriormente, este proyecto se centra en la batalla que las grandes ciudades como Bogotá le están ganando a la naturaleza por medio de la construcción masiva de diferentes estructuras urbanas, desplazando a espacios cada vez más escasos a estos ecosistemas tan necesarios para el ser humano. Por eso, y pensando en contribuir de gran manera, la propuesta realizada ha sido planteada con el ob- jetivo de incentivar a la comunidad del Área Andina a contribuir con el bienestar del medio ambiente. Por ende, se realizará una carrera mixta de atletismo, en la cual la inscripción será a través de la recolecta de un kilo de material reciclable por persona. Este será donado a una organización ambientalista con el fin de obtener semillas para plantar árboles y que la naturaleza recupere su espacio vital, beneficiando a todos los seres vivos.

\section{Antecedentes}

En la actualidad, el deporte mundial está generando demasiado daño al planeta. Es decir, las diferentes disciplinas deportivas aportan negativamente en el cambio climático. Pero, ¿hay eventos deportivos que apoyen al planeta con esta lucha? Pues bien, en este apartado se expondrán dos trabajos, los cuales serán fundamentales en la construcción y desarrollo del proyecto "Siembra a través de kilómetros". Lo anterior ayudará a poner en contexto los objetivos planteados y de esta manera poder asimilar las posibles dificultades que se pudieran presentar en la construcción del mismo, así como lugares vulnerables para implementarlo, tomando como factor fundamental la ayuda del deporte sobre el cambio climático. 
Primero se expondrá la investigación del autor Baquero (2017), la cual presenta la siguiente problemática:

(...) los espacios urbanos son reducidos y la calidad de estos es baja, el presente proyecto se plantea como la proyección de un espacio verde lúdico y recreativo que contribuya a la integración y relación entre diferentes espacios deportivos y recreativos, con el objetivo de consolidar las actividades del sector el Salitre y Galerías, específicamente en la zona donde se encuentra la Unidad Deportiva el Campín y el Parque el Lago.

Este proyecto busca implementar un proyecto urbano a través de espacios verdes con actividades lúdicas y recreativas, con el fin de complementar y articular los espacios públicos del sector, tomando como base el sistema ambiental existente, el cual se tendrá que modificar para lograr integrar los puntos de interés: recreativos, deportivos y culturales, así como promover la valoración frente al paisaje cultural.

Por otro lado, se integra el trabajo de investigación de Leal (2018), el cual, aunque no sea deportivo, muestra gran similitud con los objetivos y temáticas del proyecto de "siembra a través de kilómetros", pues plantea lo siguiente:
Los bosques urbanos relucen como una solución para mitigar el cambio climático en las grandes urbes. Más allá de su capacidad para aumentar la calidad de vida de las personas por sus probados beneficios en el mejoramiento del aire y la absorción de $\mathrm{CO} 2$, estos ordenan el territorio y permiten la interacción de la ciudadanía con el medioambiente. (p. 2)

Este proyecto muestra la gran debilidad y poca preocupación de la capital colombiana (Bogotá) por sus espacios verdes y su naturaleza, entrando en conflicto con la ciudadanía, la cual se muestra inconforme por el acelerado impacto negativo en sus bosques, humedales y diferentes espacios de esparcimiento, recreación y deporte.

\section{Marco teórico}

\section{Cambio climático}

Este fenómeno natural es causado por las diferentes actividades del ser humano, las cuales aumentan las emisiones de gases a la atmósfera. Por ende, se da el efecto invernadero, al igual que la retención de calor y otras variaciones en el clima. Es decir, este fenómeno está provocando que la temperatura media del planeta aumente y se produzca lo que popularmente se conoce como calentamiento global. Esto se puede evidenciar 
en el humo y los gases que expulsan los camiones, carros, motos, buses y el sistema de transporte masivo.

Partiendo de lo anterior, puede decirse que la principal causa del cambio climático es el calentamiento global, el cual tiene múltiples consecuencias negativas en los sistemas físicos, biológicos y humanos, entre otros efectos. Uno de estos efectos es la variación en el calentamiento y enfriamiento de la tierra, reacciones que en otras ocasiones se habían generado pero de forma natural. Anteriormente, estos ciclos habían sido mucho más lentos, ya que necesitaron millones de años para que ocurrieran. En la actualidad, y gracias a la actividad humana, se están alcanzando niveles que en otras épocas trajeron extinción en diferentes especies apenas en doscientos años. Esto quiere decir que la humanidad es la responsable frente a las catástrofes que ocurren hoy en día, ya que no hay conciencia, ni preocupación por los daños y complicaciones que está sufriendo el planeta.

\section{Tala de árboles en Bogotá}

Los seres humanos cada día usan más los recursos naturales de forma desmesurada e inconsciente. En la actualidad ya no hay un límite cuando se habla de generar ganancias con los re- cursos que se tienen a disposición, ejemplo de esto es la tala de árboles. Cifras del Banco Mundial del 2006 indican que en Colombia el $42 \%$ de la madera que se explota, transporta y comercializa en el país es ilegal, cosa que es realmente preocupante, ya que el trabajo de los árboles es darles más vida a las ciudades y evitar los cambios climáticos o el calentamiento global. Sin embargo, Miguel Pacheco, especialista forestal del Fondo Mundial para la Naturaleza en Colombia (WWF, por sus siglas en inglés), se atreve a señalar que "la cifra en la actualidad se queda corta, debido a que según estudios elaborados por la propia entidad señalan que la cantidad se aproxima al 75 $\%$ ". Estas son cifras realmente preocupantes porque, como se mencionaba anteriormente, los árboles son importantes en la vida de cada uno de los humanos, ya que el árbol produce oxígeno y tan solo un árbol puede absorber hasta 22 kilos de CO2 al año. Es decir, un árbol no solo evita el calentamiento global, un árbol también da vida y protección. Por ejemplo, protege a los seres vivos de algo muy peligroso hoy en día, es decir de la radiación solar.

Según Ávila (2016) del periódico El Tiempo, "El negocio de la tala ilegal de madera se traga, lentamente, a los bosques tropicales de Colombia. Cada año 
en el país, por la deforestación clandestina, se pierden unas 48.000 hectáreas de bosque, un poco más del área urbana de Bogotá". Este es uno de los grandes retos de Colombia: combatir los delitos contra el medio ambiente. Por esta razón se realiza una alianza entre diferentes organizaciones y el gobierno nacional, gracias al pacto intersectorial en pro de la madera legal. En la actualidad, este proyecto fortalece y actualiza las regulaciones para evitar que se siga comercializando la madera de forma irregular, y mejorar así el aprovechamiento planificado de los bosques.

En definitiva, el pacto que se realizó está en proceso de acordar acciones para establecer protocolos, tanto en los sectores públicos como privados, para la compra responsable de madera y sus productos, todo con el fin de evitar la tala ilegal diaria y casos extremos que se presentan por causa del calentamiento global.

\section{Temperaturas altas y gases tóxicos}

El aumento de la temperatura de la Tierra está desencadenando problemas graves en todos los ecosistemas que la componen. Debido a este problema es que están en peligro los bosques, y un ejemplo de ello son los diferentes in- cendios forestales que se originan alrededor del planeta. Estos son el pulmón del mundo, ya que son los que generan el oxígeno necesario para los seres vivos. Otro factor negativo relevante a causa de las altas temperaturas, son las inundaciones que se producen a diario en diferentes partes del mundo debido al derretimiento de los polos y el aumento en el nivel de los mares y ríos.

Se trae a relucir el tema de las emisiones de gases causados por los diferentes medios de transporte. Infortunadamente, al día de hoy no existe una ley estricta o algún tipo de acuerdo con la población para limitar la salida de carros y, por esto, se ha llegado al punto en el cual el ambiente se torna tan pesado y tóxico que se hace difícil hasta la respiración.

En los alrededores de Bogotá se encuentran varias fundaciones que apoyan al medioambiente con la siembra de árboles, de plantas y con la recuperación de fauna salvaje que ha sido sacada de sus lugares natales para la comercialización. Estas fundaciones cuentan con ayudas de personas que trabajan sin un sueldo, personas que solo quieren ver que el medioambiente vuelva a ser lo que llegó a ser hace muchos años. No obstante, ellos también requieren de donaciones de personas que no pueden 
ayudar presencialmente pero sí económicamente y empresas relativamente grandes como Foro Nacional Ambiental, Resnatur, UICN, entre otras.

\section{Resultados}

Se realizó una encuesta a la comunidad del Área Andina, con el fin de recopilar algunos datos relevantes para el desarrollo del proyecto. En este proceso se pudo determinar la participación de 20 estudiantes de las diferentes facultades de la universidad; obteniendo los siguientes datos: Ciencias de la Salud tuvo una participación del $85 \%$, el restante, equivalente al $15 \%$, se obtuvo de las facultades de Derecho, Ciencias Sociales y Humanas e Ingenierías, cada una con un $5 \%$ de participación. Además, se determinó que la población en esta oportunidad fue totalmente estudiantil, siendo 9 de estas personas empleados y 11 independientes. Se expone que el nivel de escolaridad en esta participación fue: bachiller $25 \%$; técnico $20 \%$; tecnólogo $15 \%$; y educación superior con la mayor participación la cual fue del $40 \%$.

Del total de la muestra de 20 personas, 4 encuestados tienen una edad aproximada entre 25 y 30 años; 1 persona tiene entre 31 y 40 años; y 1 persona es menor de 18 años. Por otro lado, el 70
$\%$ de la muestra de encuestados tienen aproximadamente entre 18 y 24 años. Igualmente, podemos observar en nuestros encuestados que el nivel de escolaridad del $40 \%$ de las personas tiene educación superior, el $25 \%$ son bachilleres, el $20 \%$ de los encuestados tienen técnico y finalmente el nivel escolar del $15 \%$ de la población encuestada tiene tecnólogo. Actualmente, la ocupación de nuestra población se divide entre el ser estudiante y empleado o el ser estudiante e independiente. En los resultados que nos arroja la encuesta, encontramos que 11 personas son estudiantes e independientes y, por otro lado, 9 personas se encuentran en estos momentos estudiando y con un empleo.

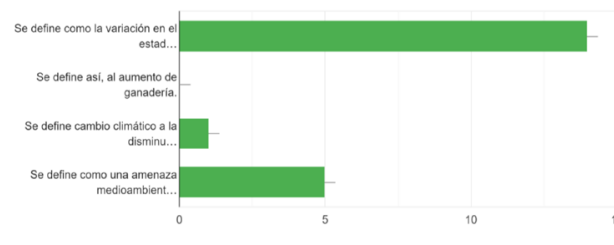

Figura 1. Definición cambio climático. Fuente: elaboración propia.

En la figura 1 se observa que del total de la muestra de 20 personas, solo el 70 $\%$ define cambio climático como la variación en el estado del sistema climático, mientras que el $25 \%$ define cambio climático como una amenaza medioambiental a la que se enfrenta la humanidad. Por 
otro lado, solo 1 persona define el cambio climático como la disminución del calor que se puede presentar en el medioambiente. El aumento de la ganadería quedó sin respuestas, lo que nos da a entender que ninguno de la población encuestada consideró que esta definición fuera acertada respecto al cambio climático.

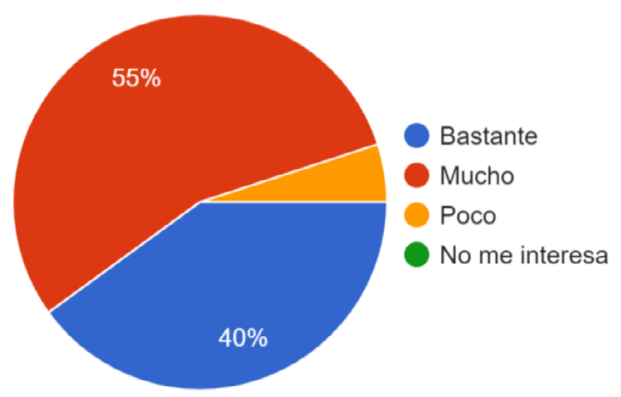

Figura 2. Preocupación por el cambio climático. Fuente: elaboración propia.

Basados en la figura 2, se muestra el gran interés de la comunidad del Área Andina por el planeta y las alteraciones negativas que produce el cambio climático. Se obtuvo $55 \%$ de población a la que le interesa 'mucho' este tema; un $40 \%$ 'bastante' y finalmente un $5 \%$-que corresponde a 1 persona- le importa 'poco'.

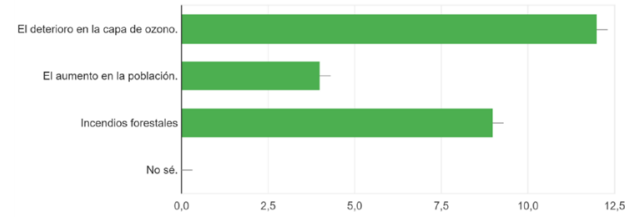

Figura 3. Impacto evidente del cambio climático. Fuente: elaboración propia.
A la pregunta formulada a los encuestados: ¿Para usted cuál es el impacto más evidente del cambio climático en la tierra?, observamos en la figura 5 que el deterioro de la capa de ozono es la opción más relevante dentro de esta, con 12 votos a favor; seguida por los incendios forestales con 9; y, de tercero, el aumento en la población con 4 votos; finalizando con la opción "no sé" con 0 votos (figura 3).

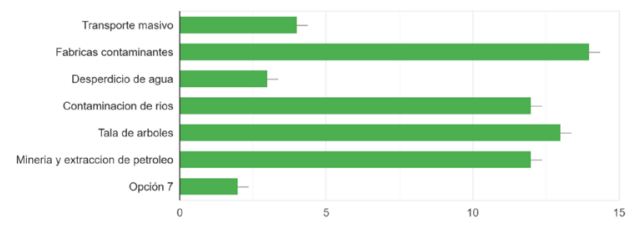

Figura 4. Motivos negativos más relevantes en el cambio climático

Fuente: elaboración propia.

Con base en la pregunta: ¿Qué motivos negativos considera usted más relevantes en el cambio climático?, se determinó que la tala de árboles junto con las fábricas contaminantes, son dos de los elementos que según la población tienen más factores negativos relevantes frente al cambio climático, lo cual hace que el proyecto sea viable por la importancia que tiene dentro de la comunidad del Área Andina (figura 4). 


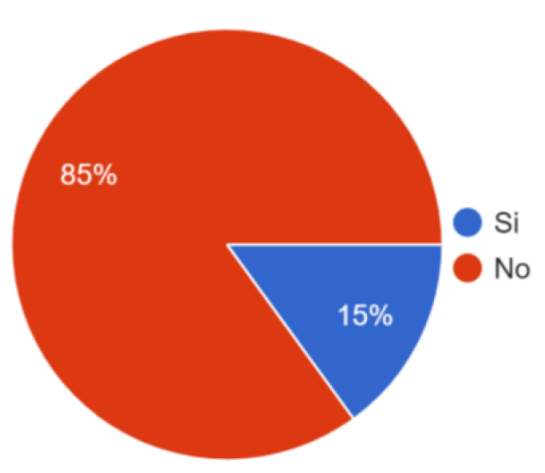

Figura 5. Conocimiento de eventos deportivos por el cambio climático.

Fuente: elaboración propia.

Con base en la pregunta: ¿Conoce algún evento deportivo que ayude al medio ambiente?, se puede evidenciar que la población del Área Andina tiene conocimiento de eventos deportivos en pro del medio ambiente. Se obtuvieron 17 respuestas positivas y 3 negativas (figura 5).

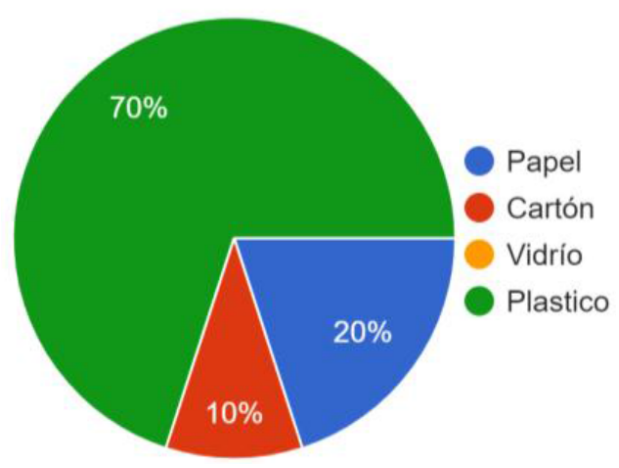

Figura 6. Material reciclado desde los hogares.

Fuente: elaboración propia.
En la figura 6, podemos observar que en la gran mayoría de los encuestados el material que más se encuentra en los hogares es el plástico, ya que casi todo está compuesto con este material, y el material que menos se produce es el vidrio. Eso es porque la gran mayoría de los productos de la canasta familiar y otras cosas son empacadas con plástico y no en vidrio.

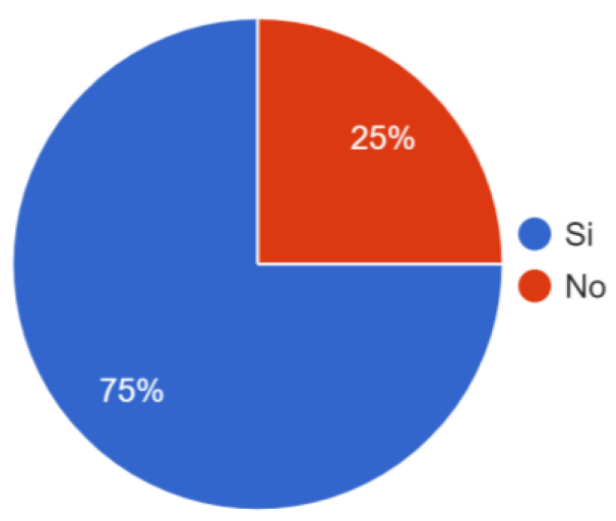

Figura 7. Siembra de árboles.

Fuente: elaboración propia.

Desde hace un par de años en los colegios e instituciones se ha venido implementado en las clases de ciencias naturales, la creación de huertas y, claro, la siembra de árboles. Por ende, no es tan difícil ahora en la población joven encontrar a personas que hayan sembrado alguna vez un árbol, de allí el porcentaje del $75 \%$ (figura 7 ). 


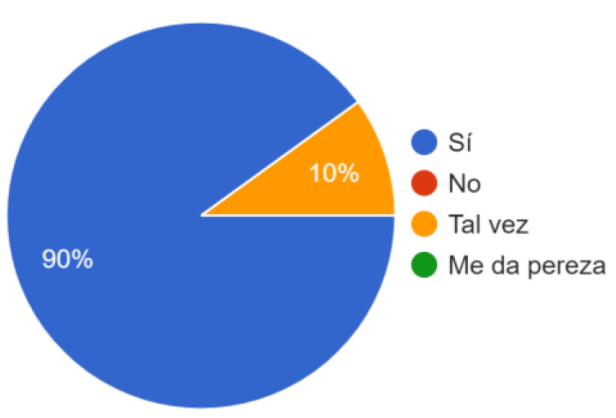

Figura 8. Participación en carrera deportiva por el cambio climático.

Fuente: elaboración propia.

En la figura 8, podemos observar que la mayoría de las personas (90\%) son solidarias, $\mathrm{y}$ tan solo por participar en una carrera y ayudar con donaciones al medioambiente la gente es feliz. Esto sería algo parecido como la Teletón, pero para árboles.

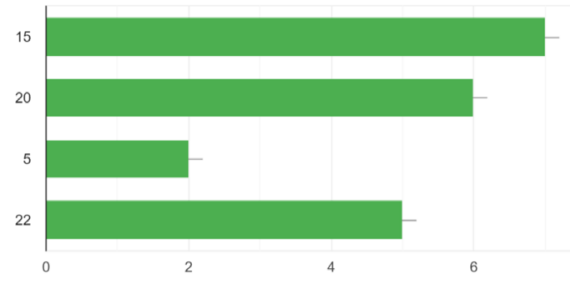

Figura 9. Participación en carrera deportiva por el cambio climático.

Fuente: elaboración propia.

A la pregunta: ¿Cuántos árboles considera usted que un ser vivo necesita para obtener el oxígeno necesario para poder vivir?, los resultados estuvieron bastante divididos y se puede evidenciar que en algunos casos adivinaron la respuesta. Muy pocos encuestados estuvieron en lo correcto gracias a la educación recibida en el bachillerato. Para que una sola persona pueda respirar, se necesitan 22 árboles (figura 9).

\section{Discusión y conclusiones}

En cuanto al tema de mejorar los problemas que se desencadenan debido al cambio climático en todo el mundo, decidimos hacer una propuesta con la cual esperábamos ayudar un poco al planeta. Sin embargo, debido a la contingencia que se presentó no pudimos realizar una investigación propiamente presencial. No obstante, gracias a esta emergencia, y vale la pena resaltar que realmente ha sido de gran ayuda para el cambio climático, pues se ha descontaminado una gran parte del planeta que ya poco a poco se estaba devastando. Si lo de la emergencia no hubiera ocurrido, en realidad llegaríamos tanto al tope de la situación del impactante cambio climático que la Tierra empezaría a responder con muchas más inundaciones de lo normal, con extinciones de animales y muchas cosas más, es decir muchas catástrofes. Por eso, esta contingencia se encuentra a favor de nuestro proyecto, ya que en lo propuesto recalcamos 
mucho la ayuda al medio ambiente $\mathrm{y}$, a pesar de no tener una investigación propiamente presencial, se logró tener una ayuda virtual, en donde los estudiantes de la Fundación Universitaria del Área Andina fueron los actores principales de nuestras encuestas, dando su opinión al respecto.

Igualmente, se pudo observar que la población se encuentra muy entusiasmada con la carrera que se realizaría por el medio ambiente, con el fin de plantar árboles y con ello ayudar al medio ambiente. Esto lo demuestran las diferentes encuestas que se realizaron a la población al azar. La población se encuentra consciente de nuestra situación crítica y, por ello, les gustaría ayudar a plantar árboles con la carrera. Sabemos que la gran mayoría ya han sembrado anteriormente un árbol. Tal vez si se hacen más carreras didácticas como esta que intentamos de realizar, la gente se hará consciente de que con solo correr en una maratón ayuda al medio ambiente, con la siembra de árboles que ayudan a que podamos respirar un aire más limpio.

\section{Referencias}

Ávila, C. (5 de noviembre de 2016). El país pierde 48.000 hectáreas de bosque al año. El Tiempo. Recuperado de: https://www. eltiempo.com/colombia/otras-ciudades/tala-de-arboles-ilegal-en-colombia-51113

Baquero, A. J. y Gil, A. M. (2017). Consolidación de las actividades del sistema ambiental entre el parque El Lago y la Unidad Deportiva El Campin (Bachelor's thesis, Universidad Piloto de Colombia).

Bellever, E. (16 de enero de 2018). La importancia del árbol. Recuperado de: https:// www.google.com/amp/s/tendenzias.com/ eco/la-importancia-del-arbol/\%3famp

Corzo, G. T. (2007). Manejo del arbolado urbano en Bogotá. Territorios, (16-17), 149173.

Leal, J. A. (2018). La planificación del arbolado urbano en Bogotá y su impacto en la calidad de vida. Recuperado de: http://hdl. handle.net/10654/17789.

Panadero, A. N. (2010). Importancia de los sistemas silvopastoriles en la reducción del estrés calórico en sistemas de producción ganadera tropical. Revista de Medicina Veterinaria, (19), 113-122. 